\title{
Scanning of the Sun and Other Celestial Bodies with Help of Gravitation Spectroscopy
}

\author{
Kristina Zubow ${ }^{1}$, Anatolij Zubow ${ }^{2}$, Viktor Anatolievich Zubow ${ }^{2 *}$ \\ ${ }^{1}$ Department of Research and Development, AIST Handels-und Consulting GmbH, Groß Gievitz, Germany \\ ${ }^{2}$ Department of Computer Science, Humboldt University Berlin, Johann von Neumann Haus, Berlin, Germany \\ E-mail:zubow@informatik.hu-berlin.de,aist@zubow.de \\ Received March 11,2011; revised April 10, 2011; accepted April 22, 2011
}

\begin{abstract}
The resonance interaction of weak gravitation radiation (WGR) from agarose hydrogel with the gravitation radiation (GR) caused by celestial bodies (Sun, Jupiter, Uranus, Mercury and Moon) has been investigated by the Zubow gravitation mass spectroscopy (ZGMS). The absorption of WGR by the Sun was found to change at the moment when the Sun appears in the slide plane of gravitation proton resonance (SPGPR, plane going through the Earth rotating axis and the sample place on the Earth surface). There were analyzed the signals of the gravitation Sun (GS), Mercury and Moon. GS contains signals of the corona, nucleus and sub-nucleus. Here the nature of the last one is near to the matter of "naked" protons in hydrogen bonds (HB) of the sensor with which it interacts. The proton model as analogous to the black hole has been proved experimentally. The sub-nucleus was concluded to be of quarks' nature but the nucleus of neutrons' one. The GR velocity in the sun system has been determined experimentally additionally, the influence of gas giants (GG) on it. At the moment of Sun and GG opposition the GR velocity was 2 - 8 times higher than that one of the light. GG reduced the GR velocity in the direction of the Sun. The role of the Earth as a gravitation mirror has been supported. Six gravitation resonator signals from Moon gravitation shots (gravitation laser) were analyzed. The GR of planets was observed to influence the energy of water cluster ensembles.
\end{abstract}

Keywords: Sun Nucleus, Quarks, Gravitation, SPGPR, Structure, Properties, Molecular Cluster, Super Light Velocity

\section{Introduction}

Even in the early nineties of the last century a group of Russian scientists under the head of professor Lavrentijev found out that a photo sensor as well as micro-organisms already react to celestial bodies, still before they appear visibly. The reasons for these phenomena weren't explained [1-4] and they were ignored by the scientific society, additionally. Using the Zubow gravitation mass spectrometer (ZGMS, earlier called as flicker noise spectroscopy) it is possible to analyze the gravitation radiation (GR) of celestial bodies in the far space and to record the GR velocity $[5,6]$.

To understand better the structure and properties of Sun GR (directed from celestial bodies to the Sun $[6,7]$ ) GR was scanned with the help of ZGMS which was the aim of the present work.

\section{Experim Entally}

Agarose hydrogel (97 wt $\%$ water) modeling the biomatrix, was chosen as investigation objects (ZGMS-sensors). Here the ZGMS-sensor was placed into the liquids the measuring procedure of ZGMS was given in the publications [8-11]. The masses of molecular clusters and their frequencies were calculated according to the Zubow equation [11] using the Zubow constant $6.4 \times 10^{-15} \mathrm{~N} / \mathrm{m}$. The long-range order in the ZGMS-sensors at the molecular cluster level was analyzed during the period when the celestial bodies appeared in SPGPR [5] in North Germany $\left(53^{\circ} 38^{\prime} \mathrm{N}\right.$ and $\left.12^{\circ} 35^{\prime} \mathrm{E}\right)$. The ZGMS-sensors were placed into an earthed iron box that was protected from noises, heat and mechanical fields at most. The box itself was in a building far from industrial centers and anthropogeneous noise sources. To understand the method some curves, that reflect the GR energy flow (sum of cleaned signals), are given in [6]. Molecular mass clusters (nuclei concentrations) in the ZGMS-sensors were found to be formed in energy clusters of sta- 
of stationary gravitation waves of the space and they reflect the state of the gravitation field in the sample space. The gravitation energy flow of celestial bodies resonates with WGR of protons in the sensor which shall be recorded. The algorithm for the signal extraction is given in [10]. To find the correct celestial bodies' position the program ZET 9 (www.astrozet.net) was applied.

\section{Results and Discussion}

In Figure 1, the ZGMS scanning results of the Sun and Mercury at the moment when they appeared in SPGPR are shown.

As visible $\mathrm{N}$ achieved exstreme values at moments when visible and gravitation signals of Sun and Mercury appeared in SPGPR. That agreed with the two-component GR structure: main gravitation wave and ripple, caused by the main wave. Here the ripple spread with light velocity [12].

To discuss the signal character in the area of gravitation Sun in detail a higher ZGMS resolution has been applied (Figure 2). According to Figure 2 the Sun GR was found to be heterogeneously and to be represented by a group of signals. Regarding the geometric parameters the visual and gravitation Sun discs were assumed to be the same. On the left and right side from the main signal there was observed a group of more weak signals. To understand the nature of these signals we analyzed the average molecular mass $\left(\mathrm{M}_{\mathrm{GMS}}\right)$ which is a more sensitive characteristic for the cluster ensemble up to 3 million Dalton (Figure 3).

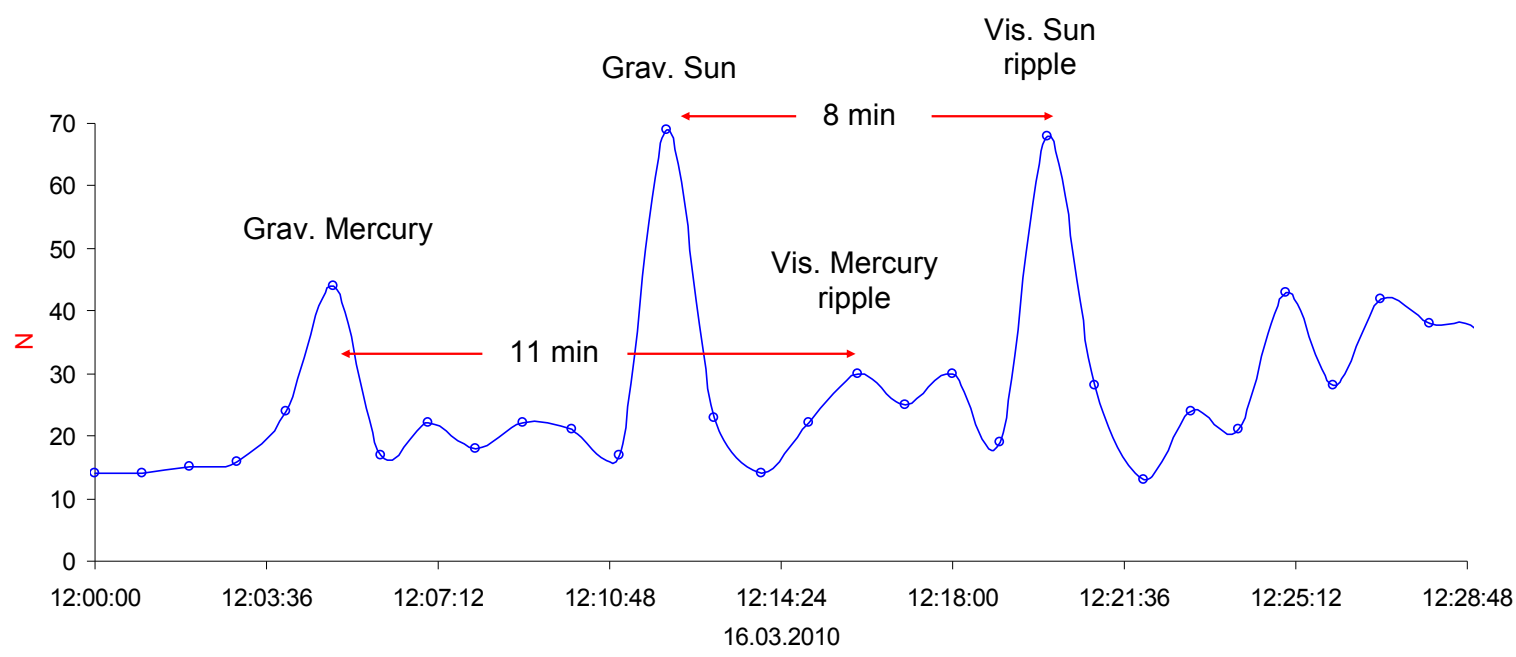

Figure 1. Reaction of the number of water cluster kinds $(\mathrm{N})$ in agarose hydrogel on GR of Sun and Mercury at their appearance in SPGPR. Distance to Sun $1.49 \times 10^{8} \mathrm{~km}$, to Mercury $2.02 \times 10^{8} \mathrm{~km}$. For a cluster mass ensemble up to $3 \mathrm{million}$ Dalton.

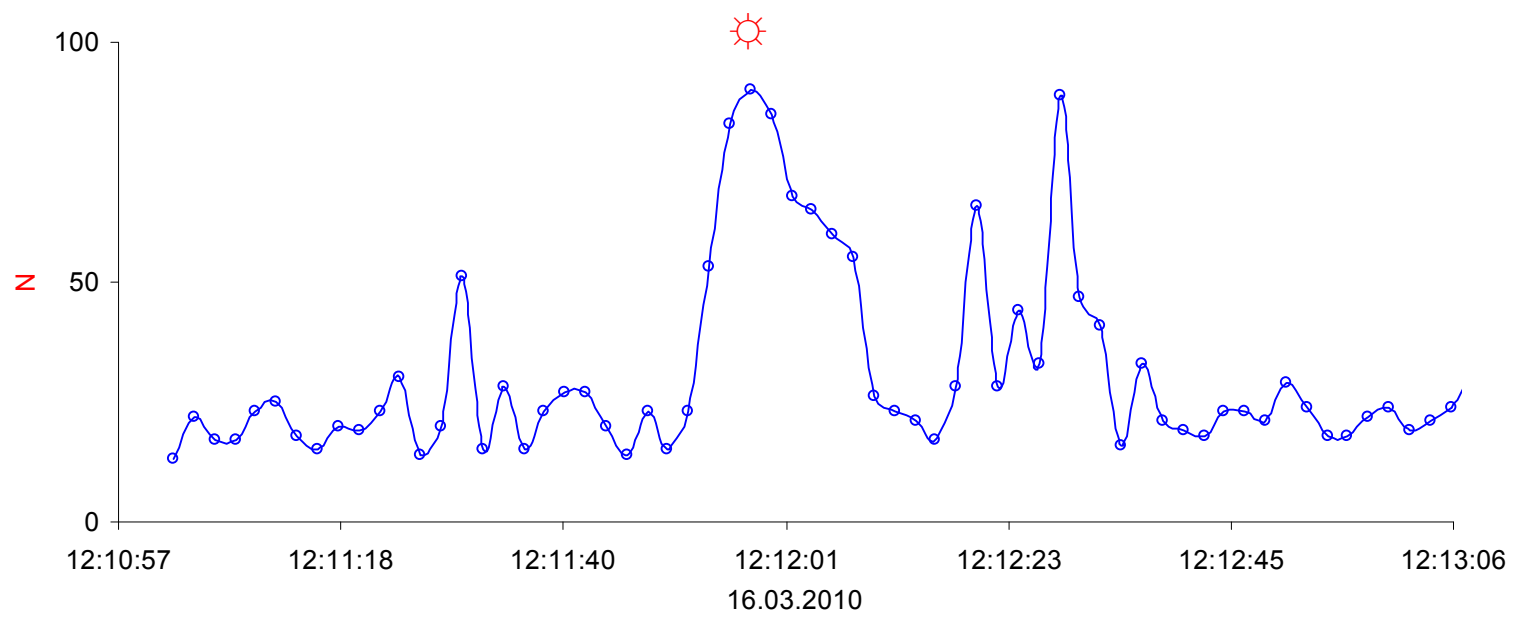

Figure 2. Signal structure in the area of the gravitation Sun (Figure 1). Scanning step of $2 \mathrm{~s}$. The center of the gravitation Sun in SPGPR is marked with 
II

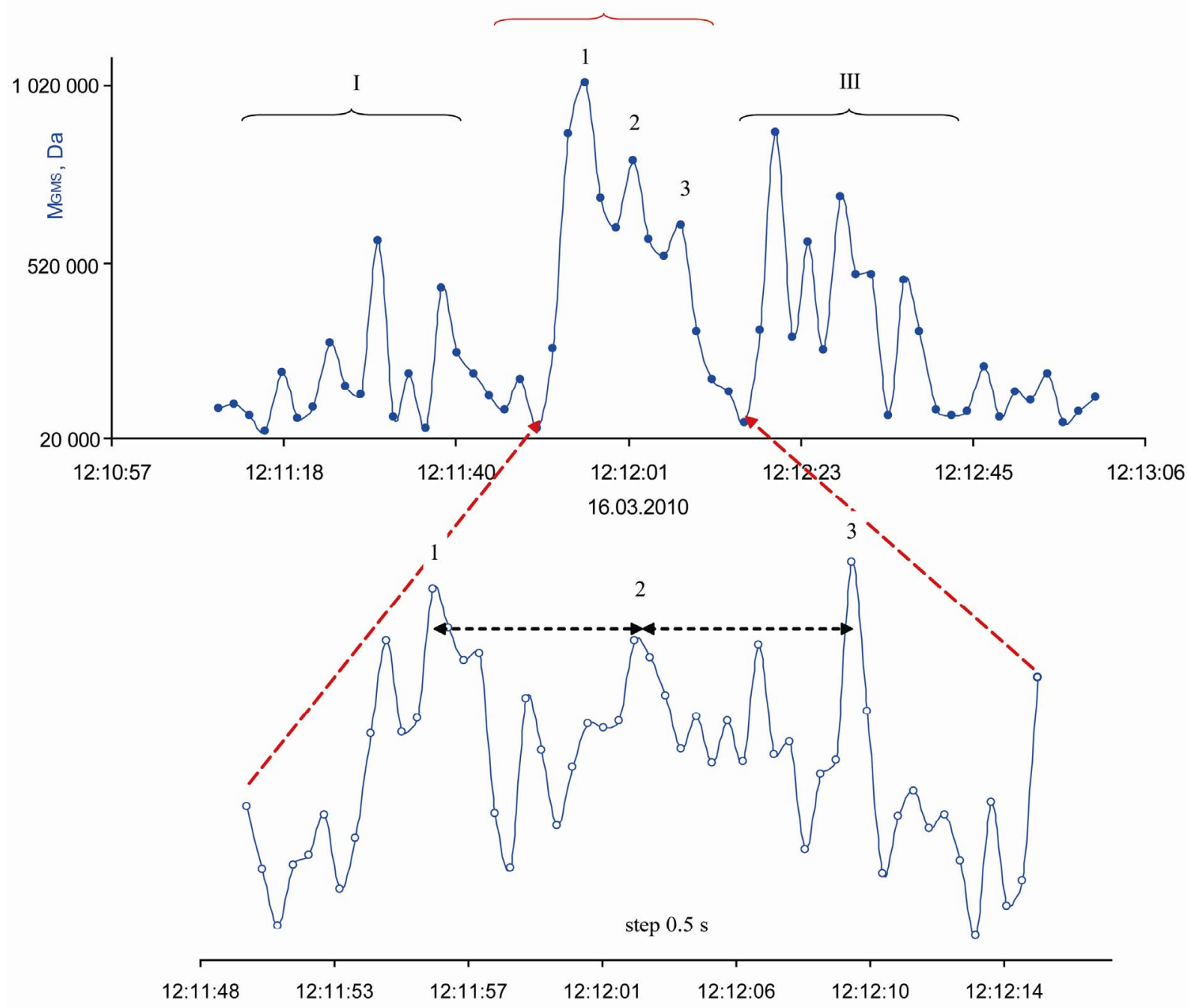

Figure 3. The change of the average molecular mass of water clusters in hydrogel at the time when signals of the gravitation Sun appeared (Figure 1). The spectrum (Figure 2) was analyzed in 2 second and 0.5 second steps.
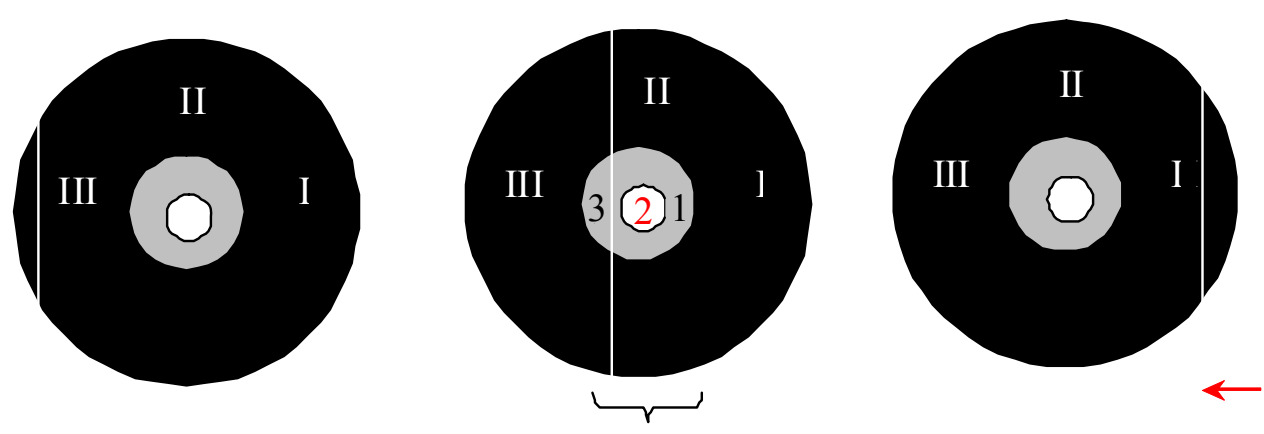

$282,100 \mathrm{~km}$

Figure 4. Model of the gravitation Sun disc, that appeared in SPGPR (white vertical line) and scanned the events in the time diapasons I, II and III (Figure 3). The horizontal arrow means the SPGPR movement. The black, grey and white discs were ascribed to the gravitation Sun, the Sun nucleus and the Sun sub-nucleus (2), accordingly. 
The $\mathrm{M}_{\mathrm{GMS}}$ value reflects the effective interaction of clusters with their surroundings [6,7], that is realized by hydrogen bonds (HB, regarding to Pauling "naked" protons [13]). This interaction is shown in Figure 4 at the moment when the gravitation Sun appeared in SPGPR (see Figure 3 too). A 50 times higher $\mathrm{M}_{\mathrm{GMS}}$ is an indication for clusters' individualization, decreasing their interaction with surroundings by intercluster HB, which disappeared practically completely at the moment 1 (diapason II). The protons of HB were dissolved in the physical vacuum and they left the sensor room in the direction to Sun $[6,7,14]$. However the moment 1 cannot be ascribed to the center of the gravitation Sun, at a higher resolution of $0.5 \mathrm{~s}$ it as well as the moment 3 became an accompanying function of the moment 2 which for its part became the central one. The position of the moments 1 and 3 to the moment 2 is nearly symmetrically being an evidence for a layer structure of the Sun nucleus (onion structure), possibly.

A better understanding of the three diapasons is easier when models shall be used (Figure 4).

The intercluster HB protons were dissolved most strongly in the diapason II [6,7] (Figure 3) where three signals were observed here. These signals could be an indication to at least one sub-center (2). The size of the center $(1+2+3)$ and sub-center (2) can be calculated roughly by applying the scanning times of the gravitation Sun. They were found to amount to 26 and 4 seconds or 282100 and $43400 \mathrm{~km}$, accordingly.

Now we shall return to Figure 3. The signals of the diapasons I and III seem to be of the same nature and they could be caused by energy fluctuations inside the nucleus. Here the nucleus' surface has to be understood as a diffuse and unstable one, however. Processes that take place in the nucleus (diapason II) precede in the diapasons I and III too, with a certain probability. Analogous processes that take place inside the nucleus (diapason II) were supported to proceed in the Sun sub-center (2) too. To be explained with that there is a resonance interaction between the HB protons of the sensor and the sub-center matter, which could be an indication that both of the same nature. Then, according to [15] and to this conclusion one can imagine the matter of the Sun sub-center as condensed quarks-gluon matter. The process of the quarks' condensation to new protons in the Sun sub-center is characterized by an exothermic effect that is sufficient for the thermonuclear synthesis of helium [14]. The fluctuation processes in the nucleus have to be understood as the result of fluctuation dynamics of gravitation flows caused by a fast changing constellation of celestial bodies as well as by events in the far space. Thus, the nucleus of the gravitation Sun was concluded to consist of neutrons (1 and 3, Figure 4). This proves sufficiently the position of quark stars as an intermediate stage from neutron stars to black holes [16].
As it was shown earlier the gravitation velocity was influenced by the constellation of celestial bodies from the far space e. g. galaxies and their centers regarding to the Sun [5]. If the light velocity from the Sun to the Earth would be taken for the velocities' calibrations then it should be possible to calculate the velocity of the main gravitation wave in the near space applying, for instance, Earth, Sun and gaseous giants. For it, the time characteristics of the gravitation Sun and the influence of gaseous giants on it shall be used. In Figure 5 it shall be shown how the energy of water cluster ensembles in agarose hydrogel behaves when the Sun and gaseous giants appear in SPGPR. Here the gaseous giants and the Sun almost lie on a line in opposition to each other: Sun- Earth-Jupiter-Uranus. As visible water cluster ensembles in the hydrogel sensor lose energy at appearance of celestial bodies in SPGPR which has to be understood as gravitation energy drain from the sensor space to giant mass concentrations in the near space $[6,7]$. These effects were characterized as endothermic ones and they support the suggested model to the HB proton dissolution in physical vacuum, therefore [14].

As shown in Figure 5 gaseous giants influence the GR velocity. Depending on the position of the observer and of other surrounding mass concentrations the GR velocity seems to change permanently in the space, therefore. Large mass concentrations in the near space were observed to destroy the main gravitation flow leading to the appearance of new GR with different velocities. Super light velocities of GR were calculated in dependence of the opposition angle of the gaseous giants Jupiter and Uranus (Figure 6). As visible in Figure 6 the curse of the curve can be described as a parable with a minimum at an angle, that doesn't correspond to $180^{\circ}$ (opposition of the Sun and planets). The minimum is shifted a little to smaller angles $\left(175-176^{\circ}\right)$ explained with that the gaseous planets, the Earth and the Sun don't lie on a line. We observed that at destroyed planets' oppositions the GR velocity was highly increased e. g. at conjugation.

The generation of GR by celestial bodies has to be understood as a process in which gravitation lasers release the energy that was accumulated by these celestial bodies. After modeling this process the Earth was found to see as a large gyroscope (Figure 7) and the atomic nuclei of the Earth as elemental gyroscopes. Here in the non-exited state, the elemental gyroscopes rotate around the own axes, that are perpendicular to the rotation axis of the Earth. On the other side, the model of the rotating proton being analogous to the modern black hole model describes the formation of three to four stationary waves (quarks, Figure 7) where the quarks' number depends on the rotation velocity of the stationary wave inside the proton [6]. The formation of stationary waves in rotating isotropic liquids is already known well [17-19], that permits to understand quarks as virtual particles or perhaps better as energy clusters. 


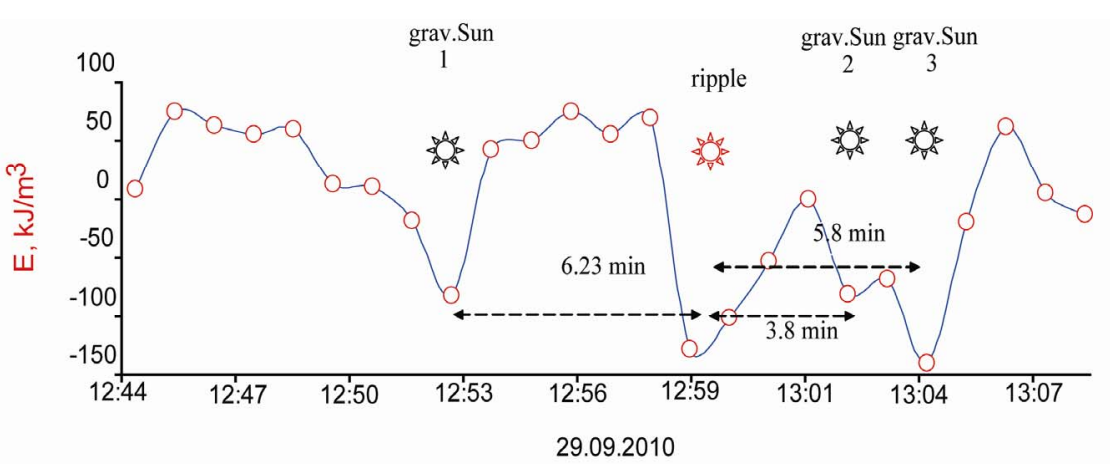

Figure 5. The influence of celestial bodies on the energy of a water cluster ensemble in agarose hydrogel (97 wt\% water, cluster ensemble mass up to 3 million Dalton). Distance to the Sun 1.00173, to Jupiter 3.96499 and to Uranus 19.09932 a.u. Gravitation velocity: main gravitation wave $1.15 \times 10^{9} \mathrm{~m} / \mathrm{s}$ (gravitation Sun 1), gravitation ripple $3 \times 10^{8} \mathrm{~m} / \mathrm{s}$ (visible Sun, light velocity), gravitation Sun 2 (GR inhibited by Jupiter) $2.06 \times 10^{8} \mathrm{~m} / \mathrm{s}$ and gravitation Sun 3 (GR inhibited by Uranus and partially by Jupiter) $1.77 \times 10^{8} \mathrm{~m} / \mathrm{s}$. The ZGMS device was calibrated according to the evaporation energy of water at $298 \mathrm{~K}$ $\left(2.45 \times 10^{6} \mathrm{~kJ} / \mathrm{m}^{3}\right)$ at which the water cluster structure is destroyed completely.

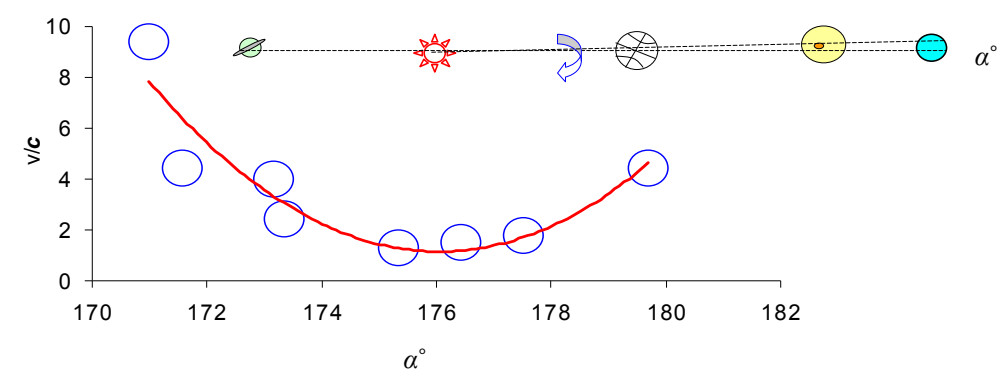

Figure 6. The influence of the opposition angle $\left(\alpha^{\circ}\right)$ of Jupiter, Uranus and Sun on the ratio of the gravitation velocity (v) to the light velocity $(c)$ (Figure 5). The results were obtained at oppositions of the Sun to gaseous giants (Neptune, Jupiter, Uranus) as well as at conjugation of Sun - Saturn between August $6^{\text {th }} 2009$ and September $29^{\text {th }} 2010$.

In this model, the protons were regarded as analogous to a "black hole" [20]. Here protons absorbed the gravitation energy from the surrounding physical vacuums after this the energy was partially concentrated and released as an energy jet [6,7]. The process strongly depended on the surroundings of the Earth and the process balance could be shifted to stronger oscillator ensembles e. g. to celestial bodies being larger than the Earth [5].

Thus, all Earth protons were activated by the rotation of the Earth where one part of the gravitation energy was released in the direction of the centrifugal force, this effect was observed at rotation of simple bodies too [21]. The second part of the gravitation energy was directed to the Earth axis and concentrated there (right model in Figure 7). With the help of this model the existence of a gravitation laser was concluded to be possible, in principle. Here for the gravitation energy supply of the laser the energy concentrated near the Earth axis as well as that one arose by the resonance interaction of sensor WGR with GR of celestial bodies in SPGPR was used. If this model is right, then gravitation laser shots have to be expected. That means we have to find experimentally signals (modes) of a gravitation resonator being typical for all laser radiations [22]. In one of our earlier works such a gravitation shot we recorded and described [23].

It should be reasonably that the GR release shall be highly increased when SPGPR hits a celestial body that appears also in the ecliptic, e.g. Jupiter, Uranus or Sun (Figure 5). Here WGR of the sensor is either weakened strongly or reinforced depending on the resonance between sensor nuclei and larger cluster dimensions of the Earth and celestial bodies in SPGPR.

The signals of the gravitation Sun (Figure 3) can be considered as gravitation resonator modes too though the gravitation shot isn't only straight but it also can be diverted by celestial bodies e.g. Moon. In Figure 8 the modes of a moon gravitation shot through the Earth (gravitation mirror) directed to the Sun on September $18^{\text {th }} 2010$ are given. As shown the signal of the gravitation Sun appears eight minutes earlier in SPGPR than that one of the visible Sun. By comparing the data of the Figures 5 (gravitation energy drain) and $\mathbf{8}$ with the exo- 


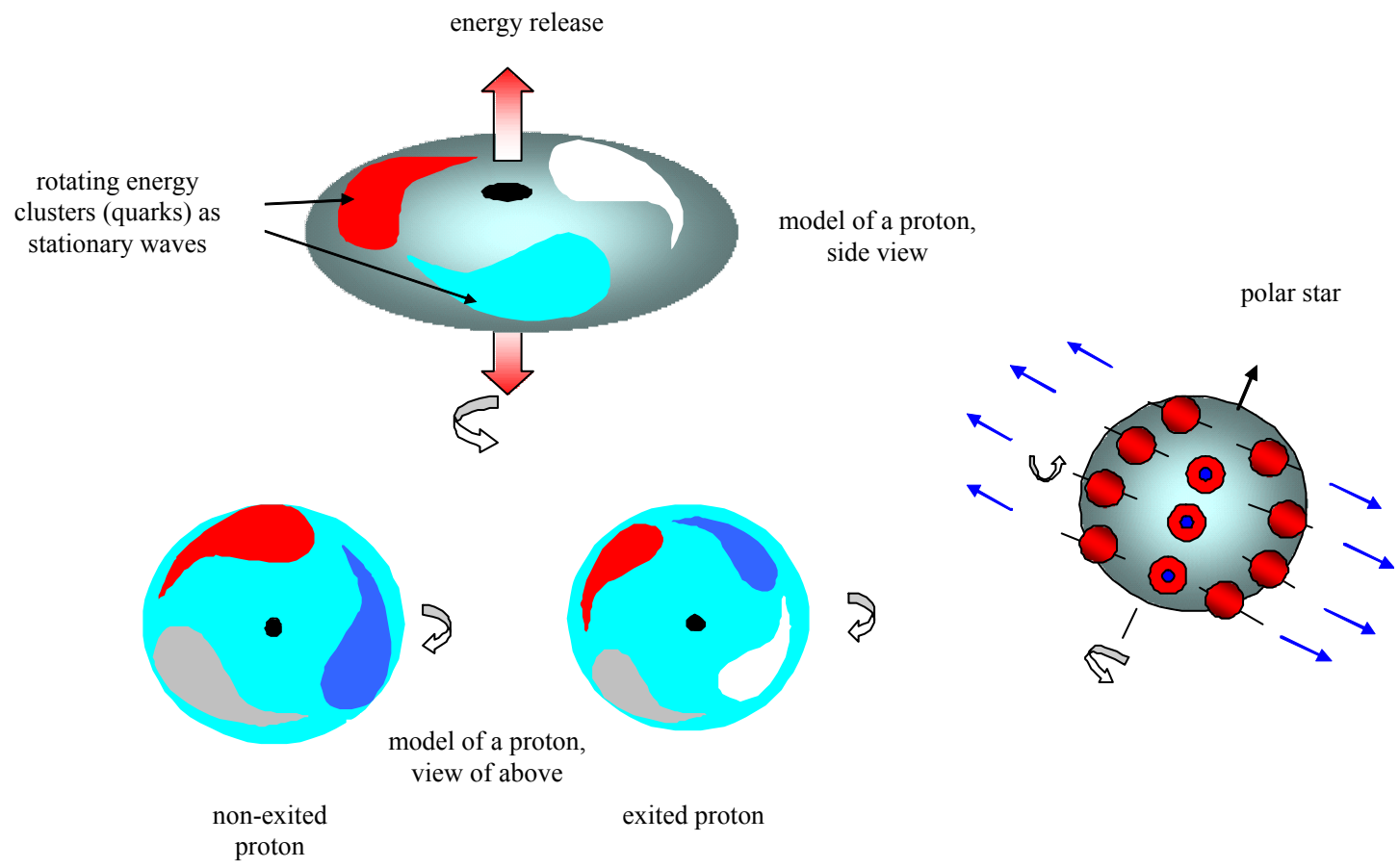

Figure 7. Structure models for exited and non-excited protons and approximate orientation of proton axes to the Earth axis (right side). The energy clusters as stationary waves rotating around the proton center (axis) are shown as colored drops. The right model discusses the Earth as a large gyroscope and the protons as elemental ones. The gravitation energy flows are marked with blue arrows and blue points.

thermic processes of the gravitation energy pumping in the sensor space the double planet Earth-Moon was found to act as a single gravitation energy donor for the Sun. Here the gravitation energy flow from the Moon is first directed to the Earth (gravitation mirror [24]) and then to the Sun. In this case the Moon was near to the ecliptic plain $\left(2^{\circ} 17 \mathrm{~min}\right)$ in which the most important gravitation events inside the sun system proceed. The energy of the modes coming from the gravitation resonator Moon (I-VI. Figure 8) was too weak to destroy the long-range order in water of the sensor explained with that the gravitation shot hits the sample only partially.

According to Ignatiev [24] the mass of the gravitation mirror amounts to $3.8 \times 10^{-3}$ of the Earth mass or $2.27 \times$ $10^{22} \mathrm{~kg}$ (for an average density of $5520 \mathrm{~kg} / \mathrm{m}^{3}$ ) and its volume - to $4.1 \times 10^{18} \mathrm{~m}^{3}$. Using a simple calculation we have shown that a paraboloid is a concave mirror formed by the earth crust of an average thickness of $33 \mathrm{~km}$ and the earth surface $\mathrm{A}=\pi \mathrm{D}^{2}$, where $\mathrm{D}=12,742 \mathrm{~km}$. Then the calculated mirror mass (hemisphere) was found to be equal to $33 \mathrm{Adx} 10^{6} / 2=4.6 \times 10^{22} \mathrm{~kg}$ and the mass of the paraboloid amounts to $2.3 \times 10^{22} \mathrm{~kg}$ [25]. The accordance of our results with those found by Ignatiev is an indication for the correctness of the proposed models (Figure 7).
As we have described in [23] gravitation shots are able to destroy computer networks and even to cause aircraft crashes. A picture of the Patomski crater in Siberia could be an example for a highly concentrated gravitation shot (http://www.panoramio.com/user/559346/tags/patomski $\% 20$ krater).

First the structure of a gravitation shot at the time when the Moon appears in the ecliptic shall be discussed. The number of water cluster kinds in agarose hydrogel at the appearance of the Moon in SPGPR and in the ecliptic of the sun system is given in Figure 9. At the moment when the satellite enters the ecliptic plane the sample protons resonate with those of the Moon and Sun attended with a highly decreased number of water cluster kinds in agarose hydrogel. It has to be mentioned that at this time the largest volcano on the Java Island and two volcanoes on Kamchatka were strongly active furthermore; strong earthquakes and tsunami in the Indonesian region (Java area) took place. Under the influence of the Moon the liquid magma seems to rise until the earth surface what therefore leads to an overheating of water in underground reservoirs [6] and connected with that to these catastrophes. The character of signals on the curve with 2 seconds' resolution is typical for a laser irradiation that can be understood as resonance modes of a gravitation resonator. 


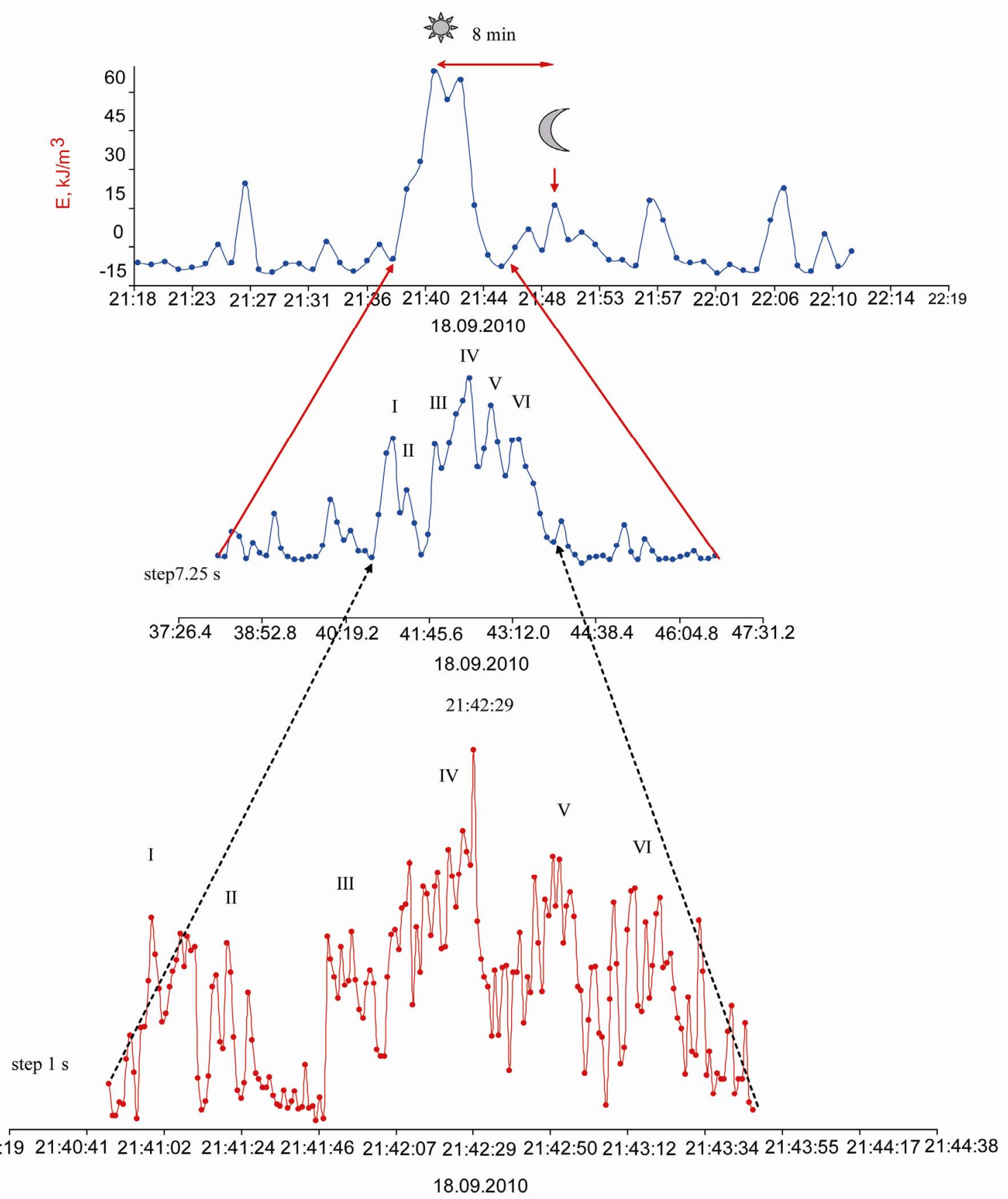

Figure 8. Gravitation shot from the Moon (geocentric latitude $2^{\circ} 17 \mathrm{~min}$ ) directed to the sensor (agarose hydrogel). Water cluster ensemble up to 3 million Dalton. I-VI modes of the gravitation resonator.

\section{Conclusions}

The Sun was found to absorb the gravitation radiation of planets surrounding it. The structure of the gravitation radiation was represented by two flows: 1) the main gravitation flow directed to the Sun and spreading out with a super-light velocity, 2) traces of the main gravitation wave as ripples with light velocity propagation. The
Sun shall be recorded by two signals, first by the signal from the gravitation Sun and second by that one from the visible Sun. The gravitation Sun is characterized by a multiple layer structure: corona, nucleus and sub-nucleus. The matter in the sub-nucleus is in resonance with that one in protons of hydrogen bonds of water in agarose hydrogel indicating that they are of similar nature (quarks-gluon matter). 


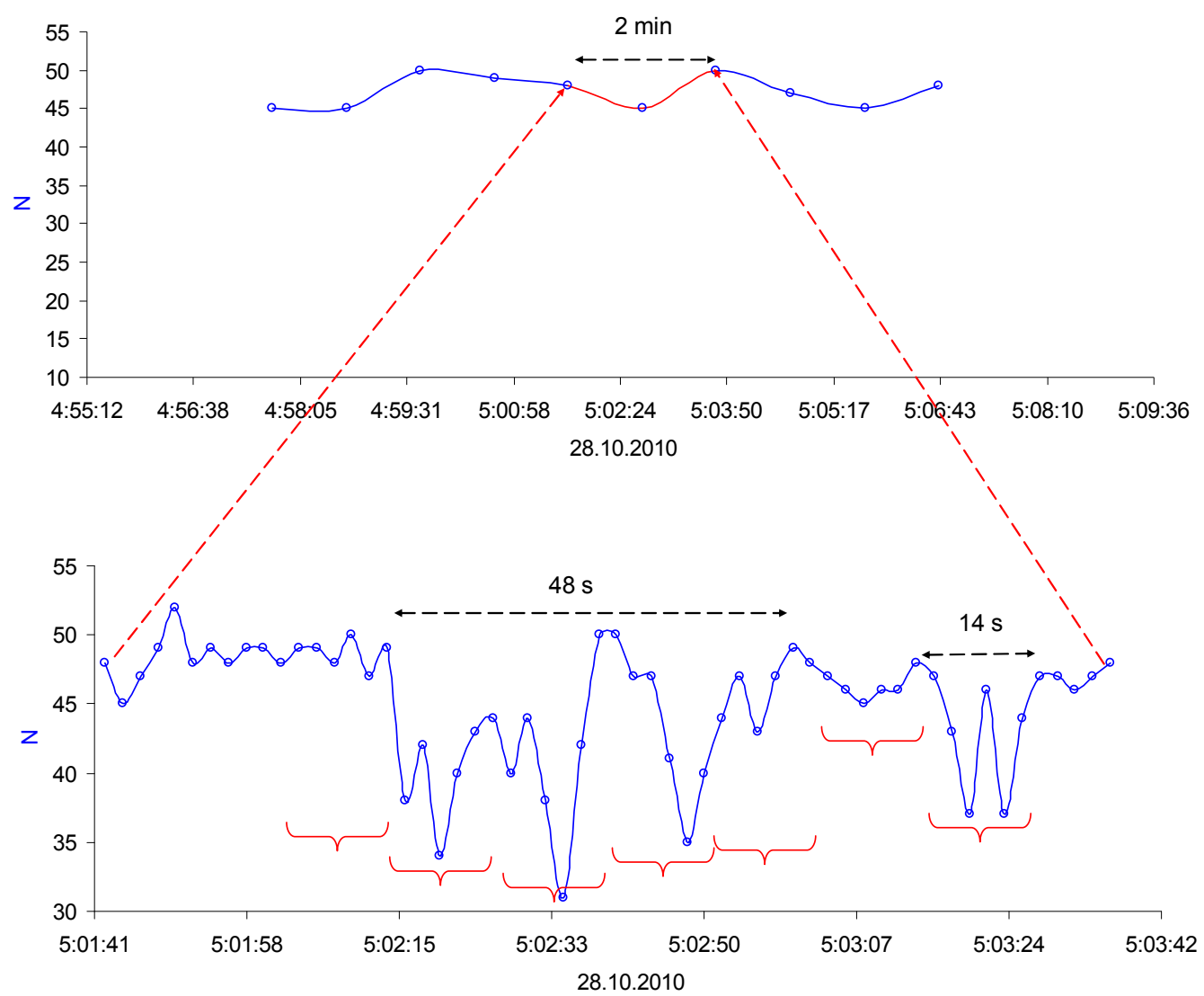

Figure 9. Change of the number of water cluster kinds in agarose hydrogel at the appearance of the Moon in SPGPR and in the ecliptic (geocentric latitude $0^{\circ} 0 \mathrm{~min}$ ). The modes of the gravitation resonator are marked with horizontal brackets.

The thermonuclear synthesis in the Sun is a secondary reaction obtaining energy from proton condensation in the sub-nucleus.

The gravitation radiation velocity in the space permanently changes depending on the observation position and on the constellation of mass concentrations regarding to the observer.

The absorption of gravitation energy by the Sun can be reinforced by celestial bodies and achieve a level of gravitation shots with catastrophic consequences.

\section{Acknowledgements}

The authors gratefully acknowledge the Aist Handelsund Consulting $\mathrm{GmbH}$ for financial support, the group of astrophysicists of professor Vasiljev S. A. (Greece) and professor Smirnov V. N. (Russia) for the fruitful discussion.

\section{References}

[1] N. A. Kozyrev and V. V. Nasonova, "Displayng of Space Factories on the Earth and Stars," M., L., 1980, pp. 76-84. (in Russian).
[2] M. M. Lavrentijev, I. A. Eganova and M. K. Luzet, "About Planet Influence on a Resistor at Distance," Doklady academii nauk. Fisika, Vol. 314, No. , 1990, pp. 352-355. (in Russian)

[3] M. M. Lavrentijev, V. A. Gusev, I. A. Eganova, M. K Luzet and S. F. Fominyh, "About Registration of Real Sun Constellation," Doklady academii nauk. Fisika, Vol. 315, No. , 1990, pp. 368-370. (in Russian)

[4] M. M. Lavrentijev, I. A. Eganova, V. G. Medvedev, V. K. Oleynik and S. F. Fominyh, "About Scanning of Sky by Kozyrev Sensor," Doklady Academii Nauk. Astronomy, Vol. 323, No. , 1992, pp. 649-652. (in Russian).

[5] K. Zubow, A. V. Zubow and V. A. Zubow, "Experimental Methods for the Determination of the Super Light Velocities of the Gravitation. Nature, Structure and Properties of Gravitation Waves," In S. K. Srivastava and K. P. Sinha, Eds., Horozons of World Physics, Nova Publishers, New York, 2010. (in Print)

[6] K.V. Zubow, A. V. Zubow and V. A. Zubow, "Principles of Gravitation Spectroscopy. New Form of Molecular Matter Processes Fields," Aist Handels and Consulting $\mathrm{GmbH}$, Berlin, 2010, p. 854. electronic book www.zubow.de. (in Russian)

[7] K. V. Zubow, A. V. Zubow and V. A. Zubow, "Ensemble of Clusters-New Form of Molecular Matter, Risks and 
Chances. Zubow Equations," In: J. C. Taylor, Ed., Advances in Chemistry Research, Nova publisher, New York, Vol. 5, 2010, pp.

[8] K. V. Zubow, A. V. Zubow and V. A. Zubow, "Using of Flicker Noise Spectroscopy for Non Destroyng Analysis of Nano Structures," Zavodskaja Laboratorija. Diagnostics of Materials, Vol. 74, No. 9, 2008, pp. 40-45. (in Russian)

[9] K. V. Zubow, A. V. Zubow and V. A. Zubow, "The Dynamics of Low Frequency Movements of Molecular Clusters in the Hardening Process of Epoxide Resins," Chem Promislennost Segodnja, Vol., No. 9, 2008, pp. 12-21. (in Russian)

[10] K. Zubow, A. V. Zubow and V. A. Zubow, "Water Clusters in Plants. Fast Channel Plant Communications. Planet Influence," Journal of Biophysics Chemistry, Vol. 1, No. 1, 2010, pp. 1-11. doi:10.4236/jbpc.2010.11001

[11] K. V. Zubow, A. V. Zubow and V. A. Zubow, "Cluster Structure of Liquid Alcohols, Water and n-Hexane," Journal of Applied Spectroscopy, Vol. 72, No. 3, 2005, pp. 321-328. doi:10.1007/s10812-005-0077-6

[12] Kokkotas, Kostas D, "Gravitational Waves," Acta Physica Polonica, Vol. 38, No. 12, 2007, pp. 3891-3923.

[13] L. Pauling, P. Pauling, W. H. Chemistry, Freeman and Company, San Francisco, 1975.

[14] K. Zubow, A. V. Zubow and V. A. Zubow, "The Phenomenon of Proton Dissolving in Vacuum and of Proton Condensation from Vacuum. Two Forms of Protons, Structure of Nuclei, Electrons and Atoms," Journal of Modern Physics, Vol. 1, No. 1, 2010 pp. 175-184. doi:10.4236/jmp.2010.13026

[15] S. Klaus, L. Stefan and S.-B. Jurgen, "Neutron Stars and Quark Phases in the Nambu-Jona-Lasinio Model," Physical Review C: Nuclear Physics, Vol. 60, No. 2, 1999, pp.025801/1-025801/11.

[16] K. Nakazato, K. Sumiyoshi and S. Yamada, "Astrophysical Implications of Equation of State for Had-
ron-Quark Mixed Phase: Compact Stars and Stellar Collapses," Physical Review D: Particles, Fields, Gravitation, and Cosmology, Vol. 77, No. 10, 2008, pp. 103006/1$103006 / 12$

[17] M. van Dyke, An Album of Fluid Motion, The Parabolic Press, Stanford California, 1982, p. 83.

[18] S. N. Dikarev, "Laboratory Study of Resonant Regimes of Motions of Homogeneous Fluid with Free Surface in a Tilted Rotating Container," Izvestiâ Akademii nauk SSSR. Fizika atmosfery $i$ okeana, Vol. 26, No. 9, 1990, pp. 982-992. (in Russian).

[19] D. Yu. Manin and Yu. L. Chernousko "An Experimental Study of the Stability of a Quasi Two-Dimensional Jet Flow Produced in Rotating Fluid by Sinks and Souces," Izvestiâ Akademii nauk SSSR. Fizika atmosfery i okeana, Vol. 26, No. 5, 1990, pp. 483-492, (in Russian).

[20] V. S. Leonow, "Discoveryof Gravitation Waves by Professor M. Weinik," Agroprogress, 2001.

[21] K. Zubow, A. V. Zubow and V. A Zubow, "Phenomenon of Fast Rotate Bodies Influence on the Long-Range Order in Water," Izwestia VUZOV. Fizika., Vol. , No., 2010, (in Print, in Russian).

[22] M. E. Zabotinsky, In M. Slowar, Ed., Fisitsheskij Enziclopeditsheskij: Sovijet Enciclopedia, 1984, p. 339.

[23] K. Zubow, A. V. Zubow and V. A. Zubow, "Experimental Platform for the Investigation of the Structural Heterogeneity of the Physical Vacuumm, Vacuum Energy Risks and Chances," In V. R. Frignanni, Ed., Horozons of World Physics, Nova Publishers, New York, 2011. (In Print)

[24] A. Yu. Ignatiev, R. R. Volkas, "Geophysical Constraints on Mirror Matter within the Earth," Physical Review D: Particles and Fields, Vol. 62, No. 2, 2000, pp. 023508/1023508/7.

[25] V. N. Rogdestwensky, In M. Slowar, Ed., Fisitsheskij Enziclopeditsheskij, Sovijet Enciclopedia, 1984, p. 200. 